\title{
Isolated medial patellofemoral ligament reconstruction for recurrent patellofemoral instability: analysis of outcomes and risk factors
}

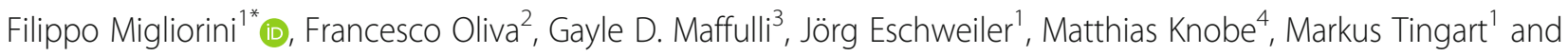
Nicola Maffulli2,5,6

\begin{abstract}
Background: The medial patellofemoral ligament (MPFL) is always damaged after patellar dislocation. In selected patients, MPFL reconstruction is necessary to restore a correct patellar tracking. Despite the large number of different techniques reported to reconstruct the MPFL, there is no consensus concerning the optimal procedure, and debates is still ongoing. The present study analysed the results after isolated MPFL reconstruction in patients with patellofemoral instability. Furthermore, a subgroup analysis of patients presenting pathoanatomical risk factors was made.

Methods: In November 2020, the main electronic databases were accessed. All articles reporting the results of primary isolated MPFL reconstruction for recurrent patellofemoral instability were considered for inclusion. Only articles reporting a minimum 12-month follow-up were eligible.

Results: Data from a total of 1777 knees were collected. The mean age of the patients involved was $22.8 \pm 3.4$ years. The mean follow-up was $40.7 \pm 25.8$ months. Overall, the range of motion $(+27.74 ; P<0.0001)$ and all the other scores of interests improved at last follow-up: Kujala $(+12.76 ; P=0.0003)$, Lysholm $(+15.69 ; P<0.0001)$, Tegner score $(+2.86 ; P$ $=0.006$ ). Seventy-three of 1780 patients (4.1\%) showed a positive apprehension test. Thirty of 1765 patients (1.7\%) experienced re-dislocations, while 56 of 1778 patients (3.2\%) showed persisting joint instability. Twenty-five of 1786 patients (1.4\%) underwent revision surgeries.
\end{abstract}

Conclusion: Isolated MPFL reconstruction for recurrent patellofemoral instability provides reliable surgical outcomes. Patients with pathoanatomical predisposing factors reported worse surgical outcomes.

Keywords: Patellofemoral instability, MPFL reconstruction, Risk factors

\footnotetext{
* Correspondence: migliorini.md@gmail.com

'Department of Orthopaedics, RWTH Aachen University Clinic, Pauwelsstraße 30, 52074 Aachen, Germany

Full list of author information is available at the end of the article
}

C C The Author(s). 2021 Open Access This article is licensed under a Creative Commons Attribution 4.0 International License, which permits use, sharing, adaptation, distribution and reproduction in any medium or format, as long as you give appropriate credit to the original author(s) and the source, provide a link to the Creative Commons licence, and indicate if changes were made. The images or other third party material in this article are included in the article's Creative Commons licence, unless indicated otherwise in a credit line to the material. If material is not included in the article's Creative Commons licence and your intended use is not permitted by statutory regulation or exceeds the permitted use, you will need to obtain permission directly from the copyright holder. To view a copy of this licence, visit http://creativecommons.org/licenses/by/4.0/. The Creative Commons Public Domain Dedication waiver (http://creativecommons.org/publicdomain/zero/1.0/) applies to the data made available in this article, unless otherwise stated in a credit line to the data. 


\section{Introduction}

Recurrent patellofemoral instability is common, accounting for approximately one-third of all knee injuries in sports medicine $[1,2]$. Patients suffering from recurrent patellofemoral instability frequently present underlying pathoanatomical abnormalities which predispose them to patellar dislocation [3, 4]. These alterations incorporate bony conformation abnormalities including trochlear dysplasia [5], lower limb mal-alignment syndromes such as tibial extra-rotation [6] and soft tissue abnormalities such as patella alta [7]. Most patients present with a combination of two or more concomitant pathoanatomical risk factors [8, 9]. Given its multifactorial aetiology, the management of recurrent patellofemoral instability can be challenging [10-12]. In non-surgical treatment, most patients experience recurrent patella dislocations, pain and instability in the affected knee [13, 14], leading to a lower level of activity and reduced quality of daily living [15]. Hence, surgical reconstruction of the medial patellofemoral ligament (MPFL) represents a feasible option in those patients $[9,16,17]$. MPFL reconstruction shows an appreciable improvement in quality of life and recreational participation $[18,19]$. As a result of the highly promising outcomes recorded, an isolated MPFL reconstruction can be performed even in patients presenting with low- to moderate-grade pathoanatomical alteration, avoiding bony interventions [20-22]. The number of different procedures described to reconstruct the MPFL in these patients is increasing exponentially, and assessment of these options has become a point of considerable research interest [23, 24]. However, there has been no consensus concerning results, and debates are still ongoing [25-27].

Thus, we conducted a systematic review of the literature to analyse results after isolated MPFL reconstruction in patients with recurrent patellofemoral instability. Furthermore, we performed subgroup analyses of patients presenting pathoanatomical risk factors. The focus of the present systematic review was on clinical scores and examinations, rate of revision surgeries, re-dislocations and persistent joint instability. We hypothesised that this procedure provides reliable surgical outcomes and that patients with predisposing risk factors are more prone to complications.

\section{Materials and methods}

\section{Search strategy}

A comprehensive review of the literature was performed according to the Preferred Reporting Items for Systematic Reviews and Meta-Analyses guidelines (PRISMA) [28]. To guide the search, a preliminary protocol was defined:

- Population: recurrent patellofemoral instability;

- Intervention: primary isolated MPFL reconstruction;
- Outcomes: clinical scores, physical examination, complications;

- Timing: $>24$ months follow-up.

\section{Literature search}

Two independent reviewers $(* ; * ; *)$ performed the search separately. The following electronic databases were accessed: PubMed, Medline, Embase and Google Scholar. In November 2020, the following terms were used in combination: knee, patellofemoral, dislocation, recurrent, instability, medial patellofemoral ligament, MPFL, tear, rupture, surgery, reconstruction, TT-TG, trochlear, dysplasia, patella alta, apprehension test, Kujala, Lysholm, Tegner, re-dislocation, failure, reoperation, revision, subluxation. If title and abstract matched the topic, the full-text was accessed. Furthermore, a cross reference of bibliographies was performed to improve the studies for inclusion. Disagreements between the authors were debated and solved by a third author $(* *)$.

\section{Eligibility criteria}

All the articles treating primary isolated MPFL reconstruction for recurrent patellofemoral instability were considered for inclusion. According to the authors' capabilities, articles published in English, French, German, Italian or Spanish were considered. Articles with level of evidence I to IV, according to the Oxford Centre of Evidence Based Medicine [28], were considered. Technical articles, comments, letters, editorials, protocols, guidelines and review articles were excluded. Biomechanical, animal and cadaveric studies were also excluded. Studies on MPFL reconstruction after total knee arthroplasty were excluded, as were articles reporting surgical outcomes regarding combined interventions were also rejected. Articles reporting MPFL rupture with direct suture, ligament plastic or medial retinaculum reefing were excluded. Articles treating MPFL reconstruction in a revision setting were also excluded, along with those treating acute injuries. Only articles reporting a minimum of 12-month follow-up were included. Only articles that reported quantitative data concerning the outcomes of interest were included. Missing data pertinent to these parameters warranted exclusion from this systematic review. The same investigators screened the articles for inclusion. A cross-reference research of the selected articles was performed to identify any article omitted from the initial search.

\section{Outcomes of interest}

Two investigators $(* * * *)$ extracted the following data independently: patient demographics (author and year, number of procedures, mean age at time of surgery); mean follow-up duration; type of study. Patients clinical status has been evaluated through the analysis of three 
scores: the Kujala Anterior Knee Pain Scale [29], the Lysholm Knee Scoring Scale [30] and the Tegner Scale [31]. Range of motion (ROM) was also analysed. Postoperative complications were recorded for each publication: apprehension test, revision surgeries, re-dislocations and persistent joint instability. The latter was defined as recurrence and/or subjective sensation of subluxation or instability [32]. Furthermore, the presence of abnormal tibial tuberosity-trochlea groove distance (TT-TG) of patellar height and trochlear dysplasia was recorded.

\section{Methodological quality assessment}

For the methodological quality assessment, we used the PEDro score. Two authors independently $(* * ; * * *)$ performed the score calculation. The PEDro score analyses each included article under several items: allocation, presence of randomisation or blinding methods, clear inclusion and exclusion criteria, duration of follow-up and type of analysis. The final score ranks from 0 (poor quality) to 10 (excellent quality). Values $>6$ points are considered as acceptable.

\section{Statistical analysis}

The statistical analysis was performed through the software IBM SPSS. For continuous variables, the weighted mean differences and the standard deviation (SD) between groups were adopted. For binary variables, the odd ratio (OR) effect measure was used. The confidence interval was set at $95 \%$ in all the comparisons. Values of $P<0.05$ were considered statistically significant.

\section{Results}

\section{Literature search}

The literature search and cross-referencing resulted in a total of 1413 references, of which 411 were rejected because of duplications. Of these, another 930 were rejected or deviations from the eligibility criteria, leaving 77 publications for review. After reading the remaining full-text articles, another 17 articles were excluded, given insufficient details and/or uncertain diagnoses or outcome measures. Finally, 55 articles were included in the present analysis (Fig. 1).

\section{Methodological quality assessment}

The PEDro score evidenced several limitations. First, only $7 \%(4 / 55)$ of article were randomised studies. None of the included studies used any blinding. Strength points were the number of prospective studies and the length of follow-up provided by most studies. In total, the PEDro score resulted in $6.38 \pm 1.0$ points, attesting to the quality of this systematic review. The results of the PEDro score assigned to each study are shown in Table 1.

\section{Demographic data}

Data from a total of 1777 knees were collected. The mean age of the patients was $22.8 \pm 3.4$ years. The mean follow-up was $40.7 \pm 25.8$ months. Twenty-three of 55 articles (42\%) reported data of patients with imaging evidence of trochlear dysplasia, 7 of 55 (13\%) with elevated TT-TG and 15 of 55 (27\%) with patella alta. Further, $33.8 \%$ (606 of 1795) of procedures were performed using a single bundle patellar graft insertion, while $66.2 \%$ though a double bundle. Patellar fixation was achieved through a bone tunnel in $44.5 \%$ (837 of 1884) of procedures, suture anchors in 30.8\% (581 of 1884), soft tissue procedures $10.9 \%$ (205 of 1884), suture 23.6\% (69 of 1884), Endobutton 3.2\% (60 of 1884), quadriceps tendon pedicle $2.9 \%$ (55 of 1884), staple $2.4 \%$ (46 of 1884) and patellar tendon pedicle $1.6 \%$ (31 of 1884). Femoral fixation was achieved though interference screw $83.6 \%$ (1492 of 1874), anchors $4.5 \%$ (80 of 1874), staple $9.2 \%$ (70 of 1874), soft tissue procedures $2.9 \%$ (52 of 1874), bone plug $1.7 \%$ (31 of 1874), adductor pedicle $1.7 \%$ (30 of 1874) and Endobutton 1.6\% (29 of 1874). Semitendinosus was used in $37.0 \%$ (699 of 1884) of procedures, gacilis $30.5 \%$ (574 of 1884 ), synthetic $6.7 \%$ (127 of 1884), quadriceps $2.9 \%$ (55 of 1884), patellar $31 \%$ (1.6 of 1884) and adductor magnus 30\% (1.6 of 1884). The demographic data of studies included are shown in Table 1.

\section{Clinical findings}

Overall, the ROM $(+27.74 ; P<0.0001)$ and all the other scores of interests improved at the last follow-up: Kujala (+12.76; $P=0.0003)$, Lysholm $(+15.69 ; P<0.0001)$, Tegner score $(+2.86 ; P=0.006)$. These results are shown in detail in Table 2.

\section{Complications}

Seventy-three of 1780 patients (4.1\%) showed a positive apprehension test. Thirty of 1765 patients (1.7\%) experienced re-dislocations, while 56 of 1778 patients (3.2\%) showed persisting joint instability. Twenty-five of 1786 patients (1.4\%) underwent further revision surgeries.

\section{Subgroup analyses}

The presence of pathoanatomical risk factors do not influence the Kujala, Lysholm and Tegner scores, as did the rate of positiveness to the apprehension test (Table 3).

Studies treating patients within the normal range of TT-TG distance reported a lower rate of revision surgeries (OR: 0.09 ; 95\% CI: 0.0302 to $0.2943 ; P<0.0001$ ), redislocations (OR: 0.2 ; 95\% CI: 0.0754 to $0.3669 ; P<0.0001$ ) and persistent join instability (OR: 0.3 ; $95 \%$ CI: 0.1660 to 0.5886; $P=0.0003$ ) compared to those treating patients with an increased TT-TG. Studies treating patients with patella height within the normal range reported a lower rate of revision surgeries (OR: $0.8 ; 95 \% \mathrm{CI}: 0.1495$ to 1.6667; $P=0.04$ ), re-dislocations (OR: 0.2 ; $95 \%$ CI: 0.0514 to $0.6044 ; P=0.006$ ) and persistent joint instability (OR: 0.2 ; $95 \%$ CI: 0.0929 to $0.4825 ; P=0.0002$ ) compared to 


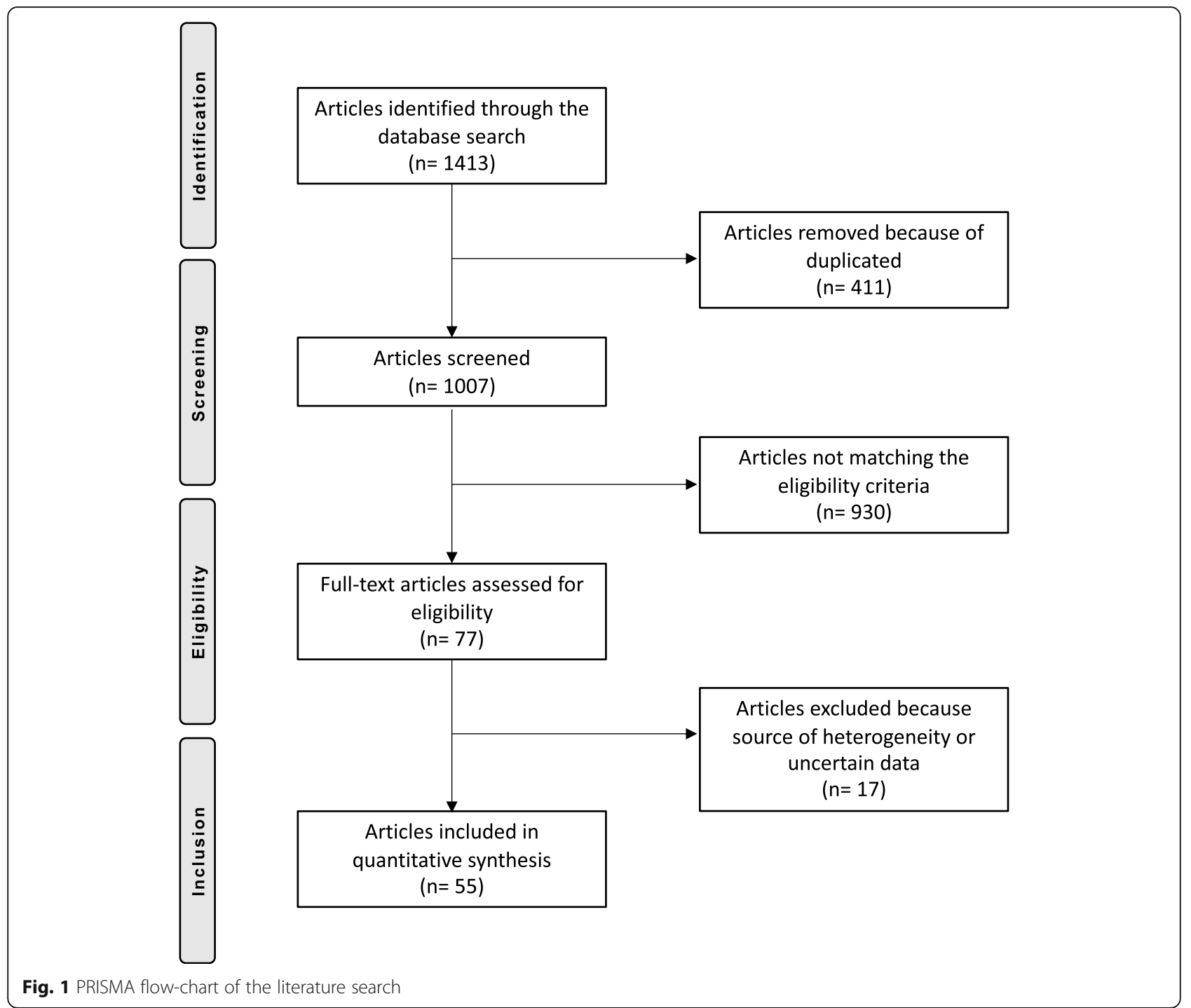

those treating patients with signs of patella alta. Studies treating patients with trochlear morphology within the normal anatomic range reported a lower rate of revision surgeries (OR: 0.2 ; $95 \% \mathrm{CI}$ : 0.0536 to $0.6541 ; P=0.009$ ), redislocations (OR: $0.2 ; 95 \% \mathrm{CI}: 0.0503$ to $0.4216 ; P=$ $0.0004)$ and persistent joint instability (OR: $0.2 ; 95 \%$ CI: 0.0832 to $0.3860 ; P<0.0001)$ compared to those treating patients with of trochlear dysplasia. These results are shown in detail in Table 4.

\section{Discussion}

The present study assessed the outcome of isolated MPFL reconstruction in selected patients with patellar instability. Isolated MPFL reconstruction for recurrent patellofemoral instability provided very good outcomes, as witnessed by the Kujala, Lysholm and Tegner scores. Patients with patella alta, trochlear dysplasia and those with elevated of TT-TG distance showed an increased rate of revision surgeries, re-dislocations and persistent joint instability compared to those without the presence of pathoanatomical risk factors. Patients with elevated TT-TG distance are more prone to revision surgery.

The MPFL is the most important static restraint to the lateral displacement of the patella during the first $30^{\circ}$ of flexion [76]. After the first patellar dislocation, the MPFL is always damaged [77, 78] and a ligament reconstruction is often required [12, 79]. Patients without imaging evidence of pathoanatomical risk factors are suitable for isolated MPFL reconstruction [80-83]. Patellar instability is a multifactorial condition [20, 84, 85]. Twenty-six percent of the patients had two, about $17 \%$ three and $15 \%$ four concomitant risk factors [86]. Other imaging studies detected similar observations $[8,9]$. Thus, proper treatment consists of adequate analysis of the associated pathoanatomical risk factors prior to MPFL reconstruction [87]. The question worth discussing remains whether 


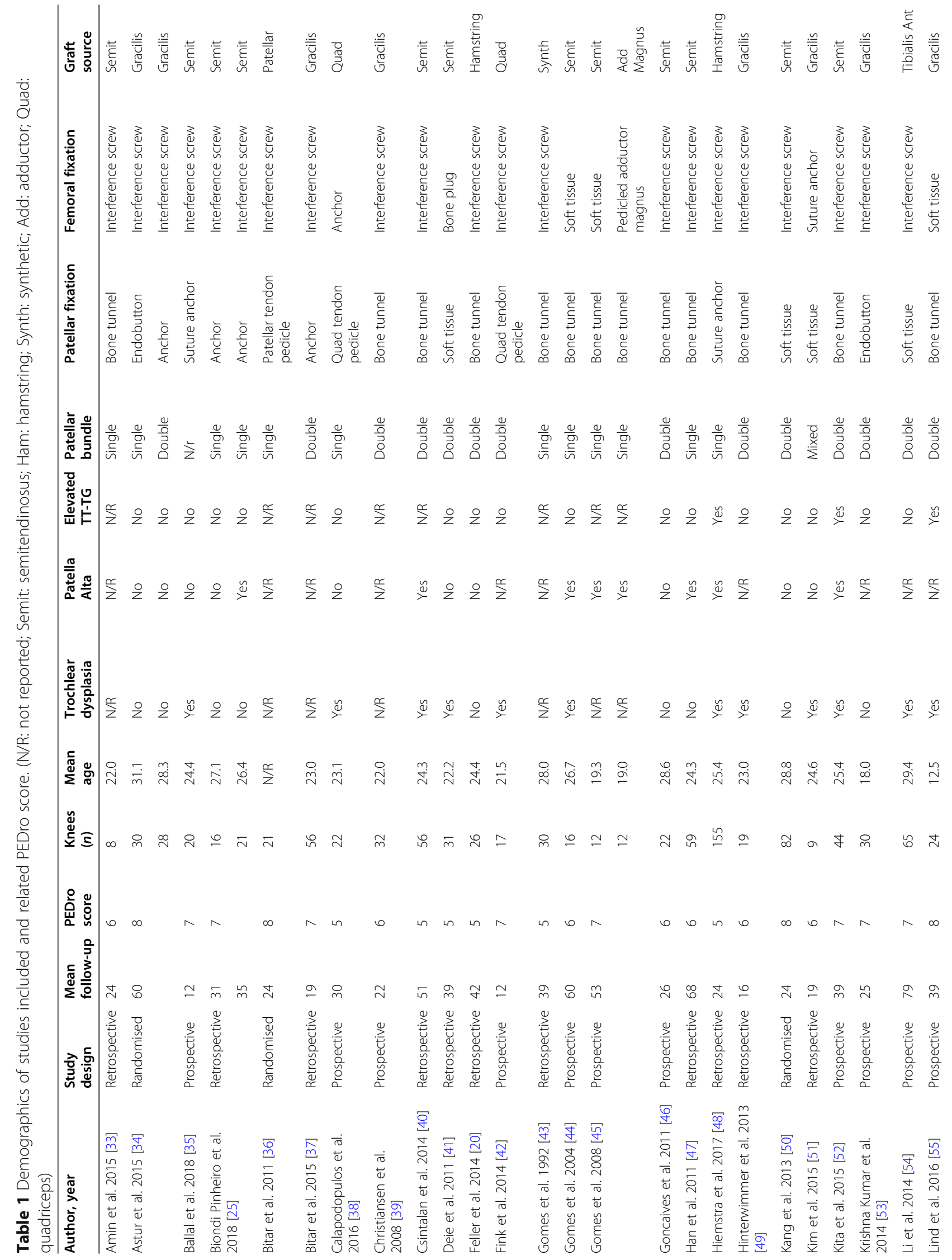




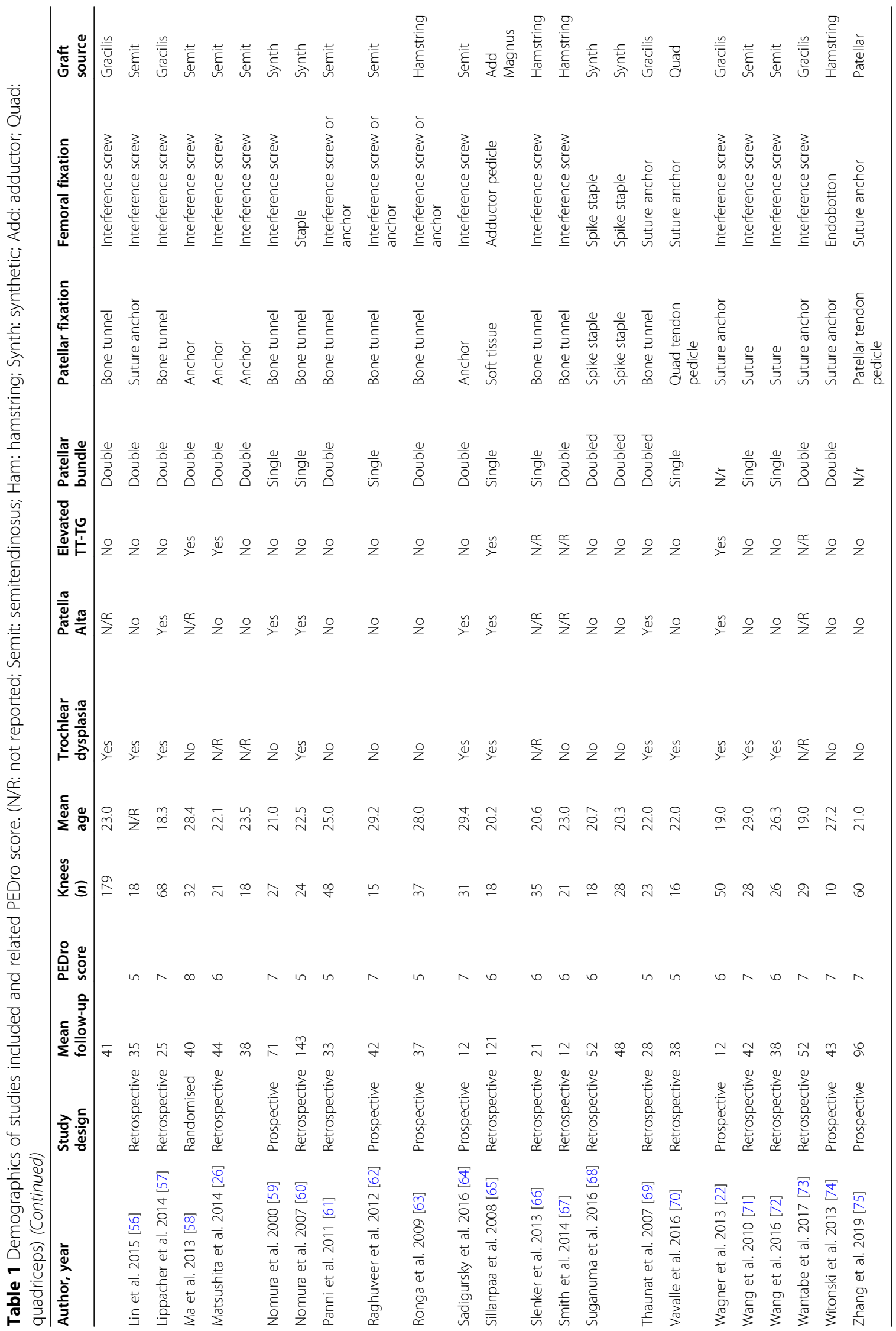


Table 2 Analyses of the endpoint: clinical scores

\begin{tabular}{lllll}
\hline Endpoint & Pre-operative & Post-operative & Improvement & P \\
\hline Kujala & $75.54 \pm 9.7(67.3$ to 81.8$)$ & $88.30 \pm 5.9(97.7$ to 71$)$ & 12.76 & 15.69 \\
Lysholm & $74.41 \pm 9.6(59.1$ to 80.4$)$ & $90.10 \pm 4.0(96.4$ to 79.7$)$ & 2.86 & $>0.0003$ \\
Tegner & $2.43 \pm 2.2(1.1$ to 3.9$)$ & $5.29 \pm 1.0(7.82$ to 4$)$ & 27.74 & 0.006 \\
Rom & $105.31 \pm 25.3(94.6$ to 118.1$)$ & $133.05 \pm 9.0(141.3$ to 125.9$)$ & 0.0001 \\
\hline
\end{tabular}

isolated MPFL reconstruction alone or combined with other procedures are needed to restore optimal biomechanics and patellar tracking [20]. Regarding preoperative planning of such procedures, no clear or detailed recommendations have been established. Each patient must be evaluated individually and the decision to combine reconstruction of the MPFL with another procedure still depends on the clinical judgement of the treating orthopaedic surgeon.

Patients presenting with high-grade of pathoanatomical risk factors are likely suitable for combined interventions. Combining different interventions aiming to stabilise the extensor mechanism most probably prevents further complications such as soft tissue damage or long-term degenerative joint disease such as osteoarthritis, while also improving quality of life and activity level $[84,88]$. In the present study, the outcomes of studies reporting the results of MPFL reconstruction in patients presenting pathoanatomical risk factors were also compared.

Analysing data from studies reporting MPFL reconstruction in patients with low-grade trochlear dysplasia showed no evidence of a statistically significant association with clinical scores or clinical examination, but nevertheless represent an increased risk to incur revision surgeries, re-dislocations and persistent joint instability. The most common intervention to restore roughly correct patellar tracking are an opening wedge osteotomy according to Albee [89] and the sulcus deepening

Table 3 Subgroup analyses of the endpoint: clinical scores

\begin{tabular}{lcll}
\hline Endpoint & Normal range & Abnormal range & $P$ \\
\hline \multicolumn{2}{l}{$\begin{array}{l}\text { Physiological } \\
\text { KT-TG }\end{array}$} & & \\
Kujala & $87.95 \pm 5.9$ & 85.328 .4 & 0.2 \\
Lysholm & $88.59 \pm 3.9$ & 86.924 .1 & 0.3 \\
$\begin{array}{l}\text { Tegner } \\
\text { Physiological patellar height }\end{array}$ & $5.58 \pm 1.1$ & 4.500 .7 & 0.1 \\
Kujala & $88.36 \pm 5.8$ & 88.125 .7 & \\
Lysholm & $88.87 \pm 3.6$ & 85.678 .4 & 0.9 \\
Tegner & $5.82 \pm 1.3$ & 4.881 .1 & 0.4 \\
Physiological trochlea morphology & & 0.3 \\
Kujala & $87.51 \pm 5.4$ & 87.318 .5 & \\
Lysholm & $91.64 \pm 4.0$ & 88.383 .9 & 0.5 \\
Tegner & $4.83 \pm 1.1$ & 5.511 .0 & 0.2 \\
\hline
\end{tabular}

trochleoplasty [90]. These procedures are associated with controversial outcomes, and performing opening wedge osteotomies must be considered with caution [91, 92]. Evidence concerning sulcus-deepening trochleoplasty is limited [93-95]. Wagner et al. [22] found worse results with isolated MPFL reconstruction in patients with severe trochlear dysplasia. Steiner et al. [96] performed an isolated MPFL reconstruction in patients with low- to severe-grade of trochlear dysplasia, and reported no dislocation at a minimum of 24-month follow-up. Recently, Kohn et al. [97] analysed the outcomes of isolated MPFL reconstruction in patients with high-grade trochlear dysplasia. The degree of trochlear dysplasia which might require an isolated MPFL reconstruction only remains unclear [97].

Studies including patients with a low grade of increased TT-TG and patella alta showed no difference in the clinical scores and clinical examination, but a moderate risk of subsequent re-dislocations and persistent joint instability. Interestingly, the analysis of the rate of revision surgeries was high in the elevated TT-TG but low in the patella alta group. These data require further investigations.

To evaluate patella alta, the most common indexes are the Caton-Deschamps [98] and/or the Install-Salvati

Table 4 Subgroup analyses of the endpoint: complications

\begin{tabular}{|c|c|c|c|}
\hline Endpoint & Odd Ratio & $95 \% \mathrm{Cl}$ & $P$ \\
\hline \multicolumn{4}{|l|}{ Physiological TT-TG } \\
\hline Apprehension & 0.9 & 0.6874 to 1.0002 & 0.8 \\
\hline Joint instability & 0.3 & 0.1660 to 0.5886 & 0.0003 \\
\hline Re-dislocations & 0.2 & 0.0754 to 0.3669 & $<0.0001$ \\
\hline Revision surgeries & 0.09 & 0.0302 to 0.2943 & $<0.0001$ \\
\hline \multicolumn{4}{|c|}{ Physiological patellar height } \\
\hline Apprehension & 0.8 & 0.7465 to 1.0432 & 0.9 \\
\hline Joint instability & 0.2 & 0.0929 to 0.4825 & 0.0002 \\
\hline Re-dislocations & 0.2 & 0.0514 to 0.6044 & 0.006 \\
\hline Revision surgeries & 0.8 & 0.1495 to 1.6667 & 0.04 \\
\hline \multicolumn{4}{|c|}{ Physiological trochlea morphology } \\
\hline Apprehension & 0.9 & 0.5230 to 1.1039 & 0.8 \\
\hline Joint instability & 0.2 & 0.0832 to 0.3860 & $<0.0001$ \\
\hline Re-dislocations & 0.2 & 0.0503 to 0.4216 & 0.0004 \\
\hline Revision surgeries & 0.2 & 0.0536 to 0.6541 & 0.009 \\
\hline
\end{tabular}


ratio [99], while a suitable method to rate the trochlear dysplasia is the classification of Dejour et al. [100]. The TT-TG distance is used to investigate the tibial extrarotation over the femoral axis [21]. These pathoanatomical risk factors with related rating index were not quantitatively evaluated by most of the included studies. Most of studies referred to low- to severe-grade of alteration, without proper data quantification. Therefore, these pathoanatomical risk factors could not be analysed in a quantitative fashion. This represents an important limitation of this study. The poor quality of most of the articles included constitutes another notable limitation. Lack of high-quality comparative trials is prevalent, therefore significantly reducing the overall evidence and opportunity to conduct a high-quality review. Surgical protocols for MPFL reconstruction and post-operative rehabilitation were not considered in the present investigation. The latter were heterogeneous throughout all the included studies. This therefore represents a potential source of bias. Indeed, approach, procedures and grafts were heterogeneous, as were the rehabilitation protocols. Given these limitations, data from the present study must be interpreted with caution. Points of strength of this systematic review are the comprehensive nature of the literature search along with the strict eligibility criteria. The methodological assessment resulted in a good quality assessment, and the baseline of samples was comparable, representing another strength point. Most studies reporting data from patients with additional previous surgeries did not clarify the nature of the interventions. Therefore, further considerations were not possible. Future studies should be aimed to clarify the role of other important risk factors, such as genu valgum, patellar dysplasia and femoral anteversion.

\section{Conclusion}

Isolated MPFL reconstruction for recurrent patellofemoral instability provides reliable surgical outcomes. Patients with low-grade patella alta, trochlear dysplasia and those with slight elevated of TT-TG distance showed an increased rate of revision surgeries, re-dislocations and persistent joint instability compared to those in whom pathoanatomical risk factors are not present.

\section{Abbreviations}

MPFL: Medial patellofemoral ligament; ROM: Range of motion; TT-TG: Tibial tuberosity-trochlea groove distance; SD: Standard deviation; OR: Odd ratio; N/R: Not reported; Semit: Semitendinosus; Ham: Hamstring; Synth: Synthetic; Add: Adductor; Quad: Quadriceps

\section{Acknowledgements}

None.

\section{Authors' contributions}

FM: literature search, data extraction, methodological quality assessment, statistical analysis manuscript draft; FO: supervision; GDM: supervision and approval; AT: manuscript draft, editing; JE: supervision and approval; MT: supervision and approval; NF: literature search, data extraction, methodological quality assessment, statistical, manuscript draft, final approval. All authors read and approved the final manuscript.

\section{Funding}

No external source of funding was used. Open Access funding enabled and organized by Projekt DEAL.

\section{Availability of data and materials}

This study does not contain any third material.

\section{Declarations}

Ethics approval and consent to participate

This article does not contain any studies with human participants or animals performed by any of the authors.

Consent for publication

All the authors approved the manuscript.

\section{Competing interests}

The authors declare that they have no competing interest.

\section{Author details}

1Department of Orthopaedics, RWTH Aachen University Clinic, Pauwelsstraße 30, 52074 Aachen, Germany. ${ }^{2}$ Department of Medicine, Surgery and Dentistry, University of Salerno, Via S. Allende, 84081 Baronissi, SA, Italy. ${ }^{3}$ Wholelife Clinics, London, UK. ${ }^{4}$ Department of Orthopedics and Trauma Surgery, Lucerne Cantonal Hospital, Lucerne, Switzerland. ${ }^{5}$ Barts and the London School of Medicine and Dentistry, Centre for Sports and Exercise Medicine, Queen Mary University of London, Mile End Hospital, 275 Bancroft Road, London E1 4DG, UK. 'SChool of Pharmacy and Bioengineering, Keele University Faculty of Medicine, Thornburrow Drive, Stoke on Trent, UK.

Received: 19 October 2020 Accepted: 24 March 2021

Published online: 06 April 2021

\section{References}

1. Halabchi F, Abolhasani M, Mirshahi M, Alizadeh Z. Patellofemoral pain in athletes: clinical perspectives. Open Access J Sports Med. 2017:8:189-203. https://doi.org/10.2147/OAJSM.S127359.

2. Migliorini F, Driessen A, Quack V, et al. Patellar fixation graft via suture anchors versus tunnel techniques during isolated MPFL reconstruction for recurrent patellofemoral instability: a systematic review of the literature. Arch Orthop Trauma Surg. 2020;140(9):1201-10. https://doi.org/10.1007/s004 02-020-03420-8

3. Migliorini F, Trivellas A, Colarossi G, et al. Single- versus double-bundle patellar graft insertion for isolated MPFL reconstruction in patients with patellofemoral instability: a systematic review of the literature. Arch Orthop Trauma Surg. 2020;140(6):769-76. https://doi.org/10.1007/s00402-020-033769.

4. Migliorini $F$, Rath $B$, Tingart $M$, et al. Surgical management for recurrent patellar dislocations in skeletally immature patients. Eur J Orthop Surg Traumatol. 2019;29(8):1815-22. https://doi.org/10.1007/s00590-019-02483-7.

5. Dejour $H$, Walch $G$, Neyret $P$, Adeleine P. Dysplasia of the femoral trochlea. Revue de chirurgie orthopedique et reparatrice de l'appareil moteur. 1990; 76(1):45-54.

6. Dejour H, Walch G, Nove-Josserand L, Guier C. Factors of patellar instability: an anatomic radiographic study. Knee Surg Sports Traumatol Arthrosc. 1994; 2(1):19-26. https://doi.org/10.1007/BF01552649.

7. Insall J, Goldberg V, Salvati E. Recurrent dislocation and the high-riding patella. Clinical orthopaedics and related research. 1972;88:67-9. https://doi. org/10.1097/00003086-197210000-00012.

8. Steensen RN, Bentley JC, Trinh TQ, Backes JR, Wiltfong RE. The prevalence and combined prevalences of anatomic factors associated with recurrent patellar dislocation: a magnetic resonance imaging study. The American journal of sports medicine. 2015;43(4):921-7. https://doi.org/10.1177/036354 6514563904.

9. Longo UG, Berton A, Salvatore G, Migliorini F, Ciuffreda M, Nazarian A, et al. Medial Patellofemoral Ligament Reconstruction Combined With Bony Procedures for Patellar Instability: Current Indications, Outcomes, and 
Complications. Arthroscopy. 2016;32(7):1421-7. https://doi.org/10.1016/j.a rthro.2016.01.013.

10. Fithian DC, Paxton EW, Stone ML, Silva P, Davis DK, Elias DA, et al. Epidemiology and natural history of acute patellar dislocation. Am J Sports Med. 2004;32(5):1114-21. https://doi.org/10.1177/0363546503260788.

11. Migliorini F, Trivellas A, Driessen A, et al. Graft choice for isolated MPFL reconstruction: gracilis versus semitendinosus. Eur J Orthop Surg Traumatol. 2020;30(5):763-70. https://doi.org/10.1007/s00590-020-02636-z.

12. Felli L, Alessio-Mazzola M, Lovisolo S, Capello AG, Formica M, Maffulli N. Anatomy and biomechanics of the medial patellotibial ligament: A systematic review. Surgeon. 2020. https://doi.org/10.1016/j.surge.2020.09.005.

13. Trikha SP, Acton D, O'Reilly M, et al. Acute lateral dislocation of the patella: correlation of ultrasound scanning with operative findings. Injury. 2003;34(8): 568-71. https://doi.org/10.1016/S0020-1383(02)00382-0.

14. Migliorini F, Driessen A, Quack V, et al. Surgical versus conservative treatment for first patellofemoral dislocations: a meta-analysis of clinical trials. Eur J Orthop Surg Traumatol. 2020;30(5):771-80. https://doi.org/10.1 007/s00590-020-02638- $\mathrm{x}$.

15. Smith TO, Donell ST, Chester $R$, et al. What activities do patients with patellar instability perceive makes their patella unstable? Knee. 2011;18(5): 333-9. https://doi.org/10.1016/j.knee.2010.07.003.

16. Migliorini F, Rath B, Tingart M, Niewiera M, Eschweiler J. Distal alignment procedures for patellofemoral instability: comprehensive review of the literature. Eur J Orthop Surg Traumatol. 2019;29(7):1579-88. https://doi.org/1 0.1007/s00590-019-02451-1.

17. Tsai CH, Hsu CJ, Hung CH, Hsu HC. Primary traumatic patellar dislocation. J Orthop Surg Res. 2012;7(1):21. https://doi.org/10.1186/1749-799X-7-21.

18. Reagan J, Kullar R, Burks R. MPFL reconstruction: technique and results. Orthop Clin North Am. 2015;46(1):159-69. https://doi.org/10.1016/j.ocl.2014.09.012.

19. Stupay KL, Swart E, Shubin Stein BE. Widespread implementation of medial patellofemoral ligament reconstruction for recurrent patellar instability maintains functional outcomes at midterm to long-term follow-up while decreasing complication rates: a systematic review. Arthroscopy. 2015;31(7): 1372-80. https://doi.org/10.1016/j.arthro.2014.12.029.

20. Feller JA, Richmond AK, Wasiak J. Medial patellofemoral ligament reconstruction as an isolated or combined procedure for recurrent patellar instability. Knee Surg Sports Traumatol Arthrosc. 2014;22(10):2470-6. https:/ doi.org/10.1007/s00167-014-3132-0.

21. Stephen JM, Lumpaopong P, Dodds AL, Williams A, Amis AA. The effect of tibial tuberosity medialization and lateralization on patellofemoral joint kinematics, contact mechanics, and stability. Am J Sports Med. 2015;43(1): 186-94. https://doi.org/10.1177/0363546514554553.

22. Wagner D, Pfalzer F, Hingelbaum S, Huth J, Mauch F, Bauer G. The influence of risk factors on clinical outcomes following anatomical medial patellofemoral ligament (MPFL) reconstruction using the gracilis tendon. Knee Surg Sports Traumatol Arthrosc. 2013;21 (2):318-24. https://doi.org/10.1 007/s00167-012-2015-5.

23. Tecklenburg K, Feller JA, Whitehead TS, et al. Outcome of surgery for recurrent patellar dislocation based on the distance of the tibial tuberosity to the trochlear groove. J Bone Joint Surg Br. 2010;92:1376-80.

24. Arendt EA, Dejour D. Patella instability: building bridges across the ocean a historic review. Knee Surg Sports Traumatol Arthrosc. 2013;21(2):279-93. https://doi.org/10.1007/s00167-012-2274-1.

25. Pinheiro Junior LFB, Cenni MHF, Nicolai OP, et al. Outcomes of medial patellofemoral ligament reconstruction in patients with patella alta. Rev Bras Ortop. 2018;53(5):570-4. https://doi.org/10.1016/j.rbo.2017.06.023.

26. Matsushita T, Kuroda R, Oka S, Matsumoto T, Takayama K, Kurosaka M. Clinical outcomes of medial patellofemoral ligament reconstruction in patients with an increased tibial tuberosity-trochlear groove distance. Knee Surg Sports Traumatol Arthrosc. 2014;22(10):2438-44. https://doi.org/10.1 007/s00167-014-2919-3.

27. Xing $X$, Shi $H$, Feng $S$. Does surgical treatment produce better outcomes than conservative treatment for acute primary patellar dislocations? A metaanalysis of 10 randomized controlled trials. J Orthop Surg Res. 2020;15(1): 118. https://doi.org/10.1186/s13018-020-01634-5.

28. Moher D, Liberati A, Tetzlaff J, Altman DG, for the PRISMA Group. Preferred reporting items for systematic reviews and meta-analyses: the PRISMA statement. BMJ. 2009;339(jul21 1):b2535. https://doi.org/10.1136/bmj.b2535.

29. Kujala UMJL, Koskinen SK, Taimela S, Hurme M, Nelimarkka O. Scoring of patellofemoral disorders. Arthroscopy. 1993;9(2):159-63. Epub 1993/01/01. https://doi.org/10.1016/S0749-8063(05)80366-4.
30. Lysholm J, Gillquist J. Evaluation of knee ligament surgery results with special emphasis on use of a scoring scale. Am J Sports Med. 1982;10(3): 150-4. https://doi.org/10.1177/036354658201000306.

31. Tegner Y, Lysholm J, Odensten M, Gillquist J. Evaluation of cruciate ligament injuries. A review. Acta Orthop Scand. 1988;59(3):336-41. https://doi.org/1 0.3109/17453678809149379.

32. Nikku R, Nietosvaara Y, Aalto K, Kallio PE. Operative treatment of primary patellar dislocation does not improve medium-term outcome: a 7-year follow-up report and risk analysis of 127 randomized patients. Acta Orthop. 2005;76(5):699-704. https://doi.org/10.1080/17453670510041790.

33. Mohamed Hamed Fahmy AHA. Minimal invasive medial patellofemoral ligament reconstruction by hamstring tendon auto-graft. Orthopedic \& Muscular System. 2015;04(01). https://doi.org/10.4172/2161-0533.1000182.

34. Astur DC, Gouveia GB, Borges $\mathrm{JH}$, et al. Medial patellofemoral ligament reconstruction: a longitudinal study comparison of 2 techniques with 2 and 5-years follow-up. Open Orthop J. 2015;9(1):198-203. https://doi.org/10.21 74/1874325001509010198.

35. Ballal $M, P V$, Ms NB. Functional outcome of Medial patellofemoral ligament injury (MPFL) reconstruction in recurrent patellar dislocation. Int J Orthop Sci. 2018;4(4):204-7. https://doi.org/10.22271/ortho.2018.v4.i4d.38.

36. Bitar AC, D'Elia CO, Demange MK, et al. Randomized prospective study on traumatic patellar dislocation: conservative treatment versus reconstruction of the medial patellofemoral ligament using the patellar tendon, with a minimum of two years of follow-up. Rev Bras Ortop (English Edition). 2011; 46:675-83.

37. Bitar AC, Demange MK, D'Elia CO, et al. Traumatic patellar dislocation: nonoperative treatment compared with MPFL reconstruction using patellar tendon. Am J Sports Med. 2012;40(1):114-22. https://doi.org/10.1177/036354 6511423742.

38. Calapodopulos CJ, Nogueira MC, Eustaquio JM, et al. Reconstruction of the medial patellofemoral ligament using autologous graft from quadriceps tendon to treat recurrent patellar dislocation. Rev Bras Ortop. 2016;51(2): 187-93. https://doi.org/10.1016/j.rbo.2015.05.002.

39. Christiansen SE, Jacobsen BW, Lund B, Lind M. Reconstruction of the medial patellofemoral ligament with gracilis tendon autograft in transverse patellar drill holes. Arthroscopy. 2008;24(1):82-7. https://doi.org/10.1016/.arthro.2007. 08.005 .

40. Csintalan RP, Latt LD, Fornalski S, Raiszadeh K, Inacio MC, Fithian DC. Medial patellofemoral ligament (MPFL) reconstruction for the treatment of patellofemoral instability. J Knee Surg. 2014;27(2):139-46. https://doi.org/10.1 055/s-0033-1360652

41. Deie M, Ochi M, Adachi N, Shibuya H, Nakamae A. Medial patellofemoral ligament reconstruction fixed with a cylindrical bone plug and a grafted semitendinosus tendon at the original femoral site for recurrent patellar dislocation. Am J Sports Med. 2011;39(1):140-5. https://doi.org/10.1177/0363 546510377436.

42. Fink C, Veselko M, Herbort M, Hoser C. MPFL reconstruction using a quadriceps tendon graft: part 2: operative technique and short term clinical results. Knee. 2014;21(6):1175-9. https://doi.org/10.1016/j.knee.2014.05.006.

43. Ellera Gomes JL. Medial patellofemoral ligament reconstruction for recurrent dislocation of the patella: a preliminary report. Arthroscopy. 1992;8(3):33540. https://doi.org/10.1016/0749-8063(92)90064-I.

44. Ellera Gomes JL, Stigler Marczyk LR, Cesar de Cesar P, et al. Medial patellofemoral ligament reconstruction with semitendinosus autograft for chronic patellar instability: a follow-up study. Arthroscopy. 2004;20(2):14751. https://doi.org/10.1016/j.arthro.2003.11.006.

45. Gomes JE. Comparison between a static and a dynamic technique for medial patellofemoral ligament reconstruction. Arthroscopy. 2008;24(4):4305. https://doi.org/10.1016/..arthro.2007.11.005.

46. Gonçaives MBJ, Júnior LHdC, Soares LFM, et al. Medial patellofemoral ligament reconstruction to treat recurrent patellar dislocation. Rev Bras Ortop (English Edition). 2011;46(2):160-4. https://doi.org/10.1016/S2255-4971(15)30233-0.

47. Han H, Xia Y, Yun X, Wu M. Anatomical transverse patella double tunnel reconstruction of medial patellofemoral ligament with a hamstring tendon autograft for recurrent patellar dislocation. Arch Orthop Trauma Surg. 2011; 131(3):343-51. https://doi.org/10.1007/s00402-010-1173-5.

48. Hiemstra LA, Kerslake S, Lafave M. Medial patellofemoral ligament reconstruction femoral tunnel accuracy: relationship to disease-specific quality of life. Orthop J Sports Med. 2017;5:2325967116687749.

49. Hinterwimmer S, Imhoff AB, Minzlaff $P$, Saier T, Rosenstiel N, Hawe W, et al. Anatomical two-bundle medial patellofemoral ligament reconstruction with 
hardware-free patellar graft fixation: technical note and preliminary results. Knee Surg Sports Traumatol Arthrosc. 2013;21(9):2147-54. https://doi.org/1 0.1007/s00167-013-2498-8.

50. Kang H, Cao J, Yu D, Zheng Z, Wang F. Comparison of 2 different techniques for anatomic reconstruction of the medial patellofemoral ligament: a prospective randomized study. Am J Sports Med. 2013;41(5): 1013-21. https://doi.org/10.1177/0363546513480468.

51. Kim TS, Kim HJ, Ra IH, Kyung HS. Medial patellofemoral ligament reconstruction for recurrent patellar instability using a gracilis autograft without bone tunnel. Clin Orthop Surg. 2015;7(4):457-64. https://doi.org/1 0.4055/cios.2015.7.4.457.

52. Kita K, Tanaka Y, Toritsuka Y, Amano H, Uchida R, Takao R, et al. Factors affecting the outcomes of double-bundle medial patellofemoral ligament reconstruction for recurrent patellar dislocations evaluated by multivariate analysis. Am J Sports Med. 2015;43(12):2988-96. https://doi.org/10.1177/03 63546515606102.

53. Krishna Kumar M, Renganathan S, Joseph CJ, Easwar TR, Rajan DV. Medial patellofemoral ligament reconstruction in patellar instability. Indian J Orthop. 2014;48(5):501-5. https://doi.org/10.4103/0019-5413.139864.

54. Li J, Li Y, Wei J, Wang J, Gao S, Shen Y. A simple technique for reconstruction of medial patellofemoral ligament with bone-fascia tunnel fixation at the medial margin of the patella: a 6-year-minimum follow-up study. J Orthop Surg Res. 2014;9(1):66. https://doi.org/10.1186/s13018-0140066-7.

55. Lind M, Enderlein D, Nielsen T, Christiansen SE, Faunø P. Clinical outcome after reconstruction of the medial patellofemoral ligament in paediatric patients with recurrent patella instability. Knee Surg Sports Traumatol Arthrosc. 2016;24(3):666-71. https://doi.org/10.1007/s00167-014-3439-X

56. Lin KY, Lu YC, Renn JH. The double-pulley technique for anatomical doublebundled medial patellofemoral ligament reconstruction. Injury. 2015;46(8): 1619-24. https://doi.org/10.1016/j.injury.2015.04.017.

57. Lippacher S, Dreyhaupt J, Williams SR, et al. Reconstruction of the medial patellofemoral ligament: clinical outcomes and return to sports. Am J Sports Med. 2014;42(7):1661-8. https://doi.org/10.1177/0363546514529640.

58. Ma LF, Wang F, Chen BC, Wang CH, Zhou JW, Wang HY. Medial retinaculum plasty versus medial patellofemoral ligament reconstruction for recurrent patellar instability in adults: a randomized controlled trial. Arthroscopy. 2013; 29(5):891-7. https://doi.org/10.1016/j.arthro.2013.01.030.

59. Nomura E, Horiuchi Y, Kihara M. A mid-term follow-up of medial patellofemoral ligament reconstruction using an artificial ligament for recurrent patellar dislocation. Knee. 2000;7(4):211-5. https://doi.org/10.1016/ S0968-0160(00)00072-7.

60. Nomura E, Inoue M, Kobayashi S. Long-term follow-up and knee osteoarthritis change after medial patellofemoral ligament reconstruction for recurrent patellar dislocation. Am J Sports Med. 2007;35(11):1851-8. https://doi.org/10.1177/0363546507306161.

61. Panni AS, Alam M, Cerciello S, Vasso M, Maffulli N. Medial patellofemoral ligament reconstruction with a divergent patellar transverse 2-tunnel technique. Am J Sports Med. 2011;39(12):2647-55. https://doi.org/10.1177/ 0363546511420079.

62. Raghuveer RK, Mishra CB. Reconstruction of medial patellofemoral ligament for chronic patellar instability. Indian J Orthop. 2012;46(4):447-54. https:// doi.org/10.4103/0019-5413.97259.

63. Ronga M, Oliva F, Longo UG, et al. Isolated medial patellofemoral ligament reconstruction for recurrent patellar dislocation. Am J Sports Med. 2009; 37(9):1735-42. https://doi.org/10.1177/0363546509333482.

64. Sadigursky D, de Melo Laranjeira MS, Nunes M, Caneiro RJF, Colavolpe PO. Reconstruction of the medial patellofemoral ligament by means of the anatomical double-bundle technique using metal anchors. Rev Bras Ortop. 2016;51(3):290-7. https://doi.org/10.1016/j.rbo.2015.07.010.

65. Sillanpaa P, Mattila VM, Visuri T, et al. Ligament reconstruction versus distal realignment for patellar dislocation. Clin Orthop Relat Res. 2008;466(6):147584. https://doi.org/10.1007/s1 1999-008-0207-6.

66. Slenker NR, Tucker BS, Pepe MD, Marchetto PA, Cohen SB. Short-/ intermediate-term outcomes after medial patellofemoral ligament reconstruction in the treatment of chronic lateral patellofemoral instability. Phys Sportsmed. 2013;41 (2):26-33. https://doi.org/10.3810/psm.2013.05.2009.

67. Smith TO, Mann CJ, Donell ST. Does knee joint proprioception alter following medial patellofemoral ligament reconstruction? Knee. 2014;21(1): 21-7. https://doi.org/10.1016/j.knee.2012.09.013.
68. Suganuma J, Mochizuki R, Sugiki T, Inoue Y, Kitamura K, Akutsu S, et al. Reconstruction of the Medial Patellofemoral Ligament Using a Synthetic Graft With Arthroscopic Control of Patellofemoral Congruence. Arthroscopy. 2016;32(11):2259-68. https://doi.org/10.1016/j.arthro.2016.02.004.

69. Thaunat M, Erasmus PJ. The favourable anisometry: an original concept for medial patellofemoral ligament reconstruction. Knee. 2007;14(6):424-8. https://doi.org/10.1016/j.knee.2007.08.008.

70. Vavalle G, Capozzi M. Isolated reconstruction of the medial patellofemoral ligament with autologous quadriceps tendon. J Orthop Traumatol. 2016; 17(2):155-62. https://doi.org/10.1007/s10195-015-0375-6.

71. Wang F, Kang HJ, Chen BC, Chen W, Su YL, Zhang YZ. Combination of medial patellofemoral ligament reconstruction with vastus medialis advancement for chronic patellar dislocation. Chin Med J (Engl). 2010. 123(21):3024-9.

72. Wang HD, Dong JT, Gao SJ. Medial patellofemoral ligament reconstruction using a bone groove and a suture anchor at patellar: a safe and firm fixation technique and 3-year follow-up study. J Orthop Surg Res. 2016; 11(1):138. https://doi.org/10.1186/s13018-016-0473-z.

73. Watanabe T, Muneta T, Ikeda H, Tateishi T, Sekiya I. Visual analog scale assessment after medial patellofemoral ligament reconstruction: with or without tibial tubercle transfer. J Orthop Sci. 2008;13(1):32-8. https://doi. org/10.1007/s00776-007-1196-0.

74. Witonski D, Keska R, Synder M, et al. An isolated medial patellofemoral ligament reconstruction with patellar tendon autograft. Biomed Res Int. 2013;2013:637678

75. Zhang L, Li Z. Long-term clinical results of double bundle reconstruction of the medial patellofemoral ligament for patellar instability. J Knee Surg. 2019; 32(02):153-9. https://doi.org/10.1055/s-0038-1636913.

76. Amis AA, Firer P, Mountney J, Senavongse W, Thomas NP. Anatomy and biomechanics of the medial patellofemoral ligament. Knee. 2003;10(3):21520. https://doi.org/10.1016/S0968-0160(03)00006-1.

77. Nomura E. Classification of lesions of the medial patello-femoral ligament in patellar dislocation. Int Orthop. 1999;23(5):260-3. https://doi.org/10.1007/ s002640050366.

78. Jiang B, Qiao C, Shi Y, Ren Y, Han C, Zhu Y, et al. Evaluation of risk correlation between recurrence of patellar dislocation and damage to the medial patellofemoral ligament in different sites caused by primary patellar dislocation by MRl: a meta-analysis. J Orthop Surg Res. 2020;15(1):461. https://doi.org/10.1186/s13018-020-01984-0.

79. Wang Q, Huang W, Cai D, Huang H. Biomechanical comparison of singleand double-bundle medial patellofemoral ligament reconstruction. J Orthop Surg Res. 2017;12(1):29. https://doi.org/10.1186/s13018-017-0530-2.

80. Song SYKI, Chang HG, Shin JH, Kim HJ, Seo YJ. Anatomic medial patellofemoral ligament reconstruction using patellar suture anchor fixation for recurrent patellar instability. Knee Surg Sports Traumatol Arthrosc. 2014; 22(10):2431-7. https://doi.org/10.1007/s00167-013-2730-6.

81. Wang FKH, Chen BC, Chen W, Su YL, Zhang YZ. Combination of medial patellofemoral ligament recon- struction with vastus medialis advancement for chronic patellar dislocation. Chin Med J (Engl). 2010;123(21):3024-9.

82. Wang JLLH, Liu YJ, et al. Reconstruction of the medial patellofemoral ligament with a suture-tie technique of patellar side fixation. Chin Med J (Engl). 2012;125(11):1884-8.

83. Yercan HSES, Okcu G, Ozalp RT. A novel technique for reconstruction of the medial patellofemoral ligament in skeletally immature patients. Arch Orthop Trauma Surg. 2011;131(8):1059-65. https://doi.org/10.1007/s004 02-011-1305-6.

84. Hawkins RJ, Bell RH, Anisette G. Acute patellar dislocations. The natural history. Am J Sports Med. 1986;14(2):117-20. https://doi.org/10.1177/036354 658601400204.

85. Schottle PBFS, Romero J. Clinical and radiological outcome of medial patellofemoral ligament reconstruction with a semitendinosus autograft for patella instability. Knee Surg Sports Traumatol Arthrosc. 2005;13(7):516-21. https://doi.org/10.1007/s00167-005-0659-0.

86. Steensen RNBJ, Trinh TQ, Backes JR, Wiltfong RE. The prevalence and combined prevalences of anatomic factors associated with recurrent patellar dislocation: a magnetic resonance imaging study. Am J Sports Med. 2015;43(4):921-7. https://doi.org/10.1177/0363546514563904.

87. Testa EA, Camathias C, Amsler F, Henle P, Friederich NF, Hirschmann MT. Surgical treatment of patellofemoral instability using trochleoplasty or MPFL reconstruction: a systematic review. Knee Surg Sports Traumatol Arthrosc. 2017;25(8):2309-20. https://doi.org/10.1007/s00167-015-3698-1. 
88. Atkin DM, Fithian DC, Marangi KS, Stone ML, Dobson BE, Mendelsohn C. Characteristics of patients with primary acute lateral patellar dislocation and their recovery within the first 6 months of injury. Am J Sports Med. 2000; 28(4):472-9. https://doi.org/10.1177/03635465000280040601.

89. F A. The bone graft wedge in the treatment of habitual dislocation of the patella. Med Rec. 1915;88:257-9.

90. Schottle PB, Fucentese SF, Pfirrmann C, et al. Trochleaplasty for patellar instability due to trochlear dysplasia: A minimum 2-year clinical and radiological follow-up of 19 knees. Acta Orthop. 2005;76(5):693-8. https:// doi.org/10.1080/17453670510041781.

91. Weiker GT, Black KP. The anterior femoral osteotomy for patellofemoral instability. Am J Knee Surg. 1997;10(4):221-7.

92. Kuroda R, Kambic H, Valdevit A, Andrish J. Distribution of patellofemoral joint pressures after femoral trochlear osteotomy. Knee Surg Sports Traumatol Arthrosc. 2002;10(1):33-7. https://doi.org/10.1007/s00167-0010256-9.

93. Duncan ST, Noehren BS, Lattermann C. The role of trochleoplasty in patellofemoral instability. Sports Med Arthrosc Rev. 2012;20(3):171-80. https://doi.org/10.1097/JSA.0b013e31826a1d37.

94. Laidlaw MS, Feeley SM, Ruland JR, Diduch DR. Sulcus-deepening trochleoplasty and medial patellofemoral ligament reconstruction for recurrent patellar instability. Arthrosc Tech. 2018;7(2):e113-23. https://doi. org/10.1016/j.eats.2017.08.061.

95. Carstensen SE, Menzer HM, Diduch DR. Patellar instability: when is trochleoplasty necessary? Sports Med Arthrosc Rev. 2017;25(2):92-9. https:// doi.org/10.1097/JSA.0000000000000150

96. Steiner TM, Torga-Spak R, Teitge RA. Medial patellofemoral ligament reconstruction in patients with lateral patellar instability and trochlear dysplasia. Am J Sports Med. 2006;34(8):1254-61. https://doi.org/10.1177/03 63546505285584.

97. Kohn LM, Meidinger G, Beitzel K, Banke IJ, Hensler D, Imhoff AB, et al. Isolated and combined medial patellofemoral ligament reconstruction in revision surgery for patellofemoral instability: a prospective study. Am J Sports Med. 2013;41(9):2128-35. https://doi.org/10.1177/0363546513498572.

98. Caton J, Deschamps G, Chambat P, Lerat JL, Dejour H. Patella infera. Apropos of 128 cases. Rev Chir Orthop Reparatrice Appar Mot. 1982;68(5): $317-25$.

99. Insall J, Salvati E. Patella position in the normal knee joint. Radiology. 1971; 101(1):101-4. https://doi.org/10.1148/101.1.101.

100. Dejour D, Le Coultre B. Osteotomies in patello-femoral instabilities. Sports Med Arthrosc Rev. 2007;15(1):39-46. https://doi.org/10.1097/JSA.0b013e31 803035ae

\section{Publisher's Note}

Springer Nature remains neutral with regard to jurisdictional claims in published maps and institutional affiliations.

Ready to submit your research? Choose BMC and benefit from:

- fast, convenient online submission

- thorough peer review by experienced researchers in your field

- rapid publication on acceptance

- support for research data, including large and complex data types

- gold Open Access which fosters wider collaboration and increased citations

- maximum visibility for your research: over $100 \mathrm{M}$ website views per year

At $\mathrm{BMC}$, research is always in progress.

Learn more biomedcentral.com/submissions 